\section{Adjustment of bias in the comparison of means}

SIR,- - The objective of many research investigations is to assess the effect of a stimulus on some quantitative response. This often entails comparing the mean value of the response variable between groups of subjects exposed to contrasting stimuli. Thus an occupational health study might be undertaken to compare the mean pulmonary function-for example, $\mathrm{FEV}_{1} / \mathrm{FVC}$ ratio between firefighters and police officers. Comparing the crude means between two or more groups, however, may give rise to a misleading impression about the effect of stimulus (chemicals inhaled during firefighting) and the response variable $\left(\mathrm{FEV}_{1} / \mathrm{FVC}\right)$, since such a comparison is potentially biased by confounding variables such as smoking and age. Similarly, a clinical trial might be carried out to compare the mean reduction of blood pressure in hypertensive subjects given either a new or established antihypertensive drug. Again, comparing the crude mean reduction is potentially biased by differences in baseline blood pressure in the two groups. ${ }^{1}$

Analysis of covariance (ANCOVA), a statistical technique for comparing means with adjustment for confounding bias, is usually the method of choice for adjusting the crude mean values. ${ }^{2-4}$ It must be noted, however, that ANCOVA is a parametric statistical method based on a mathematical model. Any mathematical model which attempts to represent reality necessarily stipulates conditions about the data. Some of the conditions stipulated by the ANCOVA model are that the relation between the covariate and the response is linear and equal in each comparison group, and that the error terms in the model are independent, normally distributed, and have the same variance. ${ }^{2}$ Hence, unless the data meet all the model assumptions (to within statistical limits), the ensuing statistical results obtained by ANCOVA may be uninformative and misleading.

An alternative statistical procedure to ANCOVA for the comparison of means with adjustment for confounding bias is the Z-score method. ${ }^{35}$ The main appeal of this essentially non-parametric method is that it stipulates only one condition about the data: the relation between the covariate and the response is the same in each comparison group-that is, there is no interaction. When the data show interaction, neither ANCOVA nor the Z-score method is applicable to analysis. ${ }^{3}$ One drawback of the Z-score method is that it accomplishes adjustment by stratification (analogous to the standardisation procedure for the comparison of rates). Consequently, the method is generally not feasible for adjustment of more than one or two confounders. In our view the Z-score method should be used when ANCOVA is clearly inappropriate for a given set of data.
We have written a computer procedure to perform
Z-score method in $\mathrm{SAS}^{6}$ for the mainframe con? puter as well as in dBase III* for the microcompute The programme listing, including a worked examplef is available on request.

Department of Community,

Occupational and Family Medicine,

National University of Singapore,

National University Hospital,

Singapore 0511

Republic of Singapore.

\section{References}

1 Lee J. A note on the comparison of group means based ot repeated measurements of the same subject. J Chron Dis 1980;33:673-5.

2 Anderson S, Auquier A, Hauck WW, et al. Statistical methods for comparative studies: techniques for bias reduction. New Yorf. Wiley \& Sons, 1980

3 Kleinbaum DG, Kupper LL. Applied regression analysis and other multivariate methods. North Scituate, Mass: Duxbury Pres 1978.

4 Lee J. Analysis of covariance by the SAS GLIM procedure. Cont? puters in biology and medicine London: Pergamon Press, 1986

5 Hamilton M, Pickering GW, Roberts JAF, et al. The aetiology ob hypertension; the arterial pressure in the general population. Clin Sci 1954;13:11-20.

$6 S A S$ user's guide: Basics. Version 5 ed. Cary NC: SAS Institu民 Inc, 1985.

*dBase III is a trademark of Ashton-Tate.

\section{Book reviews}

Environmental health criteria 59: principles for eval记 ating health risks from chemicals during infancy and early childhood: the need for a special approach. (Pp 7 㔽 Sw fr 9.) Geneva: World Health Organisation, 1986.

This short monograph is designed to draw attention to the special problems of evaluating possible risks to children and infants from environmental chemicals. Some of the more important areas examined are differences in feeding patterns of young and adult, the relatively greater surface area of the infant, and the. consequences resulting from the structural and funs tional immaturity of target organs and differences in the handling of the toxicant. The biological principles at work are explained and the book is well laced withp examples of the special problems associated with the young. Many of the examples came from heavy meto or pesticide intoxication.

In these days when returning to work shortly aftê the birth of a child is commonplace risks to childrent may arise from the mother's exposure in the worlo place to industrial chemicals, and may include tran 
fer of toxic chemicals to the child through breast milk or from contamination. The general principles in this book are as relevant to exposure to industrial chemicals as they are to exposure in the wider environment. I thought the book a timely illumination of one of the less well understood, but potentially important, areas of health hazard assessment.

P ILLING

Offshore medicine-Medical Care of Employees in the Offshore Oil Industry. 2nd ed. Edited by RAF Cox. (Pp 258; £54.00.) Berlin: Springer-Verlag.

Offshore medicine is designed to bring together in a practical manual the different facets and unique problems of providing medical care in the offshore oil and gas industry. This second edition has been revised and expanded to contain chapters covering general background information about the offshore oil industry, the legal aspects of safety, health, and welfare therein; preplacement and periodic examinations, back up services, medical care, special problems, diving, catering, and hygiene, dental problems, and the investigation of fatal and non-fatal accidents. In addition the appendices contain comprehensive listings of necessary drugs and equipment for routine and emergency use as well as detailed formats for hygiene inspections, medical data collection in diving accidents, and training programmes for offshore medics and first-aiders. Addresses and contact numbers for help in diving emergencies are provided.

The text is generally presented in an eminently readable manner with an obvious affection for the industry. The information provided is both comprehensive and authoritative; when controversy existsfor example, with respect to the use of helium and oxygen mixtures in the treatment of gas embolism and type 2 decompression sickness-the authors do not hesitate to give their opinion of its suitability while stating that unfortunately current opinion is tending to disagree because of misunderstanding of gas counter transfer.

All doctors, and many other paramedical and non-medical personnel, engaged in the offshore oil industry will find this book both interesting and useful in providing proper medical care for those people employed in a unique and often hazardous environment.

\section{P I RAFFAELLI}

Epidemiology of occupational health. Edited by $\mathbf{M}$ Karvonen and MI Mikheev. (Pp 392; Sw fr 48.) (WHO regional publications, European series No 20.) Copenhagen: WHO, 1986.

The aim of this book is to "... encourage and facili- tate sound and reasonable application of epidemiology to the identification of hazards, assessment of risks and evaluation of control measures in the working environment ...". It is a multiauthor book from the World Health Organisation, and the editors have not entirely succeeded in imposing a coherent style. It is not a textbook of occupational epidemiology. Some knowledge of both epidemiology and occupational health is assumed in most of the 18 contributions. To some extent its subject matter overlaps another WHO publication, Guidelines on Studies in Environmental Epidemiology (Environmental health criteria 27. Geneva: WHO, 1983), but this recent book is better presented and more readable.

It covers a wide range of topics. The basic toolsthe cohort, cross-sectional and case referent designsare given separate chapters, and the authors discuss the strengths and limitations of these strategies, as well as providing practical advice on the conduct of studies. Only one chapter is devoted specifically to statistical issues and this, though making good general points, does not claim to be a manual of techniques. Epidemiology, however, is intimately related to statistics, and individual chapters give details of analytical methods for specific applications. Several chapters place epidemiology in the general context of occupational health and that by Kazantzis and McDonald is of note-a review of the contribution of occupation to disease which is something of a tour de force, covering all organ systems with 120 references. There are also chapters on topics rarely discussed in any depth in general textbooks, but which are important, and not only in occupational health. These include the evaluation of control measures, accident epidemiology, screening, and the assessment of stress.

The reader will not find all of the contributions useful. Some rely heavily on unsupported, didactic statements. But most are careful, critical reviews that are worth reading. As a collection of essays on aspects of epidemiology in occupational health it will be a valued addition to the bookshelf of anyone interested, from professional epidemiologists to those who wish to learn more about the varied and important contribution of epidemiology to occupational health.

KATHERINE M VENABLES

\section{Notices}

The Health and Safety Executive Library and Information Service has indexed all articles relevant to health and safety at work in the British Journal of Industrial Medicine from 1944 to date. Approximately 1600 items are available in the HSELINE database, 